\title{
Hyperbaric oxygen (HBO) therapy for acute traumatic cervical spinal cord injury
}

\author{
S Asamoto*, ${ }^{*}$, H Sugiyama ${ }^{1}, \mathrm{H} \mathrm{Doi}^{1}, \mathrm{M} \mathrm{Iida}^{1}, \mathrm{~T} \mathrm{Nagao}^{2}$ and $\mathrm{K}$ Matsumoto ${ }^{3}$ \\ ${ }^{1}$ Department of Neurosurgery, Tokyo Metropolitan Ebara Hospital, Tokyo, Japan; ${ }^{2}$ Department of Neurology, Tokyo \\ Metropolitan Ebara Hospital, Tokyo, Japan; ${ }^{3}$ Department of Neurosurgery, Showa University, School of Medicine, \\ Tokyo, Japan
}

\begin{abstract}
Study design: A retrospective study of spinal cord injury (SCI) treated with and without hyperbaric oxygen (HBO) therapy.

Objectives: To report on the use of HBO in spinal cord injury.

Setting: Neurosurgical Unit, Tokyo, Japan.

Methods: Thirty-four cases of hyperextension spinal cord injury without bone damage and previous history of surgical intervention were divided into two groups, with (HBO) or without (non-HBO) therapy. The neurological findings at admission and their outcomes were evaluated by means of Neurological Cervical Spine Scale $(\mathrm{NCSS})^{1}$ and the average improvement rates in individual groups were compared.

Results: The improvement rate ranged from $100 \%$ to $27.3 \%$ with the mean value of $75.2 \%$ in the HBO group, while these values were $100 \%, 25.0 \%$ and $65.1 \%$ respectively in the non HBO group.

Conclusion: In the $\mathrm{HBO}$ group, the improvement rate indicated effectiveness in acute traumatic cervical spinal cord injury.

Spinal Cord (2000) 38, 538-540
\end{abstract}

Keywords: hyperbaric oxygen therapy; trauma; acute spinal cord injury

\section{Introduction}

Hyperbaric oxygen (HBO) therapy has been employed for treating acute traumatic cervical spinal cord injury in our institution. The results are reported in this paper.

\section{Patients and methods}

Among 114 patients with cervical spinal cord diseases admitted to our department from June 1994 to April 1999, 34 cases of hyperextension spinal cord injury with no bone damage were selected in order to obtain a discrete group. Among the 34 cases, 13 cases had received $\mathrm{HBO}$ therapy (HBO group) and 21 cases had no experience of HBO therapy (non-HBO group). No patient had a history of vertebral or spinal cord disease. HBO therapy was performed once a day for 10 days, as a rule under an absolute atmospheric pressure (ATA) of 2.0 for $85 \mathrm{~min}$ (increased pressure for $10 \mathrm{~min}$, ordinary pressure for $60 \mathrm{~min}$ and decreased pressure for $15 \mathrm{~min}$ ) using Type 2 apparatus (P-1000SE). In the HBO group, HBO therapy was performed within $24 \mathrm{~h}$ after onset of disease for 3-33 days, mean 12.1 days. The 13 cases in the HBO group included 10 males and three females

*Correspondence: S Asamoto, Department of Neurosurgery, Tokyo Metropolitan Ebara Hospital, 4-5-10 Higashi-Yukigaya, Ohta-Ku, Tokyo 145-0065, Japan aged $24-87$ years, mean: 60.5 years. The 21 cases in the non-HBO group consisted of 14 males and seven females aged 13-88 years, mean: 60.8 years. The neurological findings at admission and their outcomes were evaluated in all patients in each group by means of Neurological Cervical Spine Scale (NCSS) to obtain the rate of improvement. ${ }^{1}$ The mean rate of improvement was compared between the $\mathrm{HBO}$ and non-HBO groups. In addition to NCSS, the initial neurology and its outcome were also confirmed by ASIA impairment scale. $^{2}$ (Table 1A,B). In statistical analysis, the degree less than $5 \%$ was regarded as significant using Scheffe's method after ANOVA. The clinical syndromes at the time of admission to the hospital were classified mostly in line with the 'standard for neurological and functional classification of spinal cord injury' of ASIA. The transitional type is a case where the clinical syndrome after the onset of disease is clearly different from the clinical syndrome just after the admission to the hospital. All of the three cases showed a transition from central cord type to transverse type of spinal cord.

\section{Results}

The highest improvement rate was $100 \%$ and the lowest one was $27.3 \%$ with the mean rate of $75.2 \%$ in 
Table 1 Clinical information concerning 13 patients with hyperbaric oxygen (HBO) therapy (A), and 21 patients without HBO therapy (B)

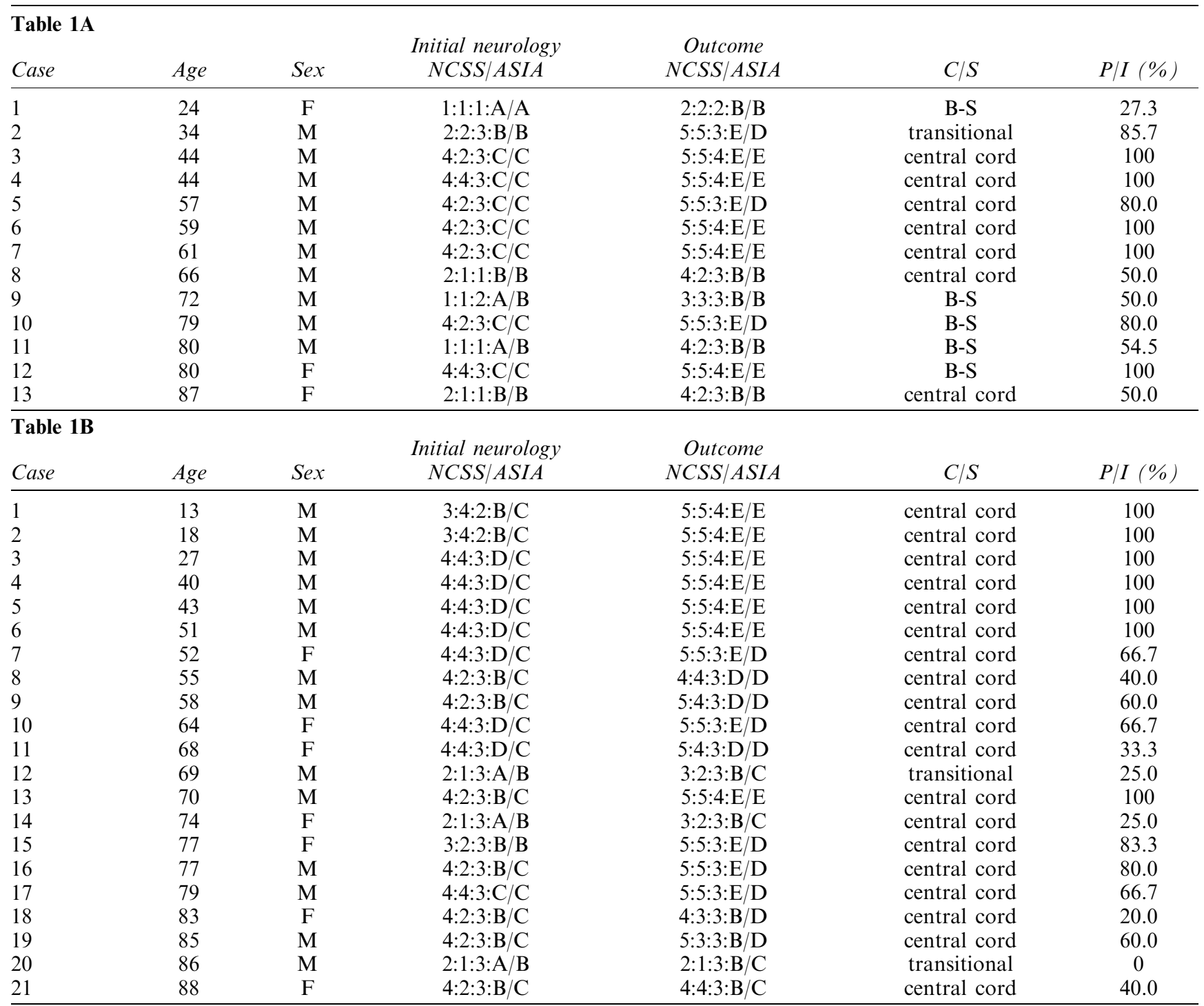

C/S: clinical syndrome; B-S: Brown-Séquard; P/I: per cent improvement

the $\mathrm{HBO}$ group, while the corresponding values in the non-HBO group were $100 \%, 25.0 \%$ and $65.1 \%$ respectively. A significant difference between the $\mathrm{HBO}$ and non-HBO groups was observed $(P<0.05)$.

\section{Discussion}

HBO therapy was started simultaneously with the opening of our hospital. Though the duration of HBO therapy was initially established by referring to the degree of symptomatic improvement only in the first three cases, the therapy was performed in accordance with our manual from the fourth case. As a result, the duration ranged from 3-33 days. However, the outcomes were all good, including the first three cases, with no difference in therapeutic efficacy.
HBO therapy is a treatment measure which is intended to restore damaged tissue by elevating oxygen levels in arterial blood in the form of dissolved oxygen through compulsive inhalation of concentrated oxygen under hyperbaric conditions and by improving the oxygen level in hypoxic tissue. Thus, HBO therapy is indicated basically for patients in a hypoxic condition. However, it is widely used not only for patients with central and/or peripheral neuropathies but also for patients in the fields of otorhinology, ophthalmology, and orthopedic surgery. ${ }^{3-7}$ Furthermore, it can be said that the effectiveness of HBO therapy is almost established against hypoxic encephalopathy. ${ }^{8,9}$ From the standpoint that the brain and spinal cord have very similar histological and physiological properties, the usefulness of $\mathrm{HBO}$ 
therapy for spinal cord injury was investigated in the present study. ${ }^{10}$ In addition, there have been some reports on the usefulness of HBO therapy not only on acute spinal cord diseases including a traumatic event reported thereinafter, of course, but also on chronic spinal cord diseases. ${ }^{11,12}$ However, concerning the usefulness of HBO therapy, it is also evident that many unclear points remain and some reports described that the final rate of improvement shows no difference from those obtained by conventional approaches. ${ }^{13}$ In the present study, the mean rate of improvement in the HBO group was superior to that in the non-HBO group.

About 20 patients with traumatic cervical cord injury are hospitalized in our hospital per year. In order to obtain patient unification, only patients who had hyperextension spinal cord injury without bone damage were included in this study because of the potential large variation due to the more frequently encountered severe cases. However, this variation distinctly existed even among patients who had hyperextension without bone damage, and there was a large range of neurological findings. Though a statistically significant difference could be observed between the $\mathrm{HBO}$ and non-HBO groups, the population examined in this study was small. The efficacy before and after HBO therapy has not been assessed by MRI.

HBO therapy is considered to be a significant subsidiary treatment. However, HBO therapy may cause some complications such as barotrauma of the ear, sinus squeeze, and pulmonary oxygen toxicity as well as explosive accident at worst. In our hospital, however, no case has suffered from such complications probably due to an adequate $24 \mathrm{~h}$ administration and treatment system by seven professional neurosurgery personnel and two clinical technicians. The optimal treatment pressure during $\mathrm{HBO}$ therapy seems to be around 2.0 ATA as regards therapeutic effects and possible burden on patients, and this is the pressure level employed in our department. However, the value of 2.5 ATA was recommended by some authors from the standpoint of oxygen diffusion into damaged nerve tissue and prevention of oxygen toxicity. ${ }^{11}$ There has been no comparative study on the therapeutic effects of 2.0 versus 2.5 ATA treatment pressure. In our hospital, 2.0 ATA is selected as a treatment pressure because (1) a therapeutically effective oxygen pressure and its concentration can be obtained even with 2.0 ATA; (2) the risk of causing complications is low compared with $2.5 \mathrm{ATA}$; and (3) establishment of increased treatment pressure takes longer.

\section{Conclusion}

HBO therapy provided a distinctly high improvement in some treated patients. The significance of $\mathrm{HBO}$ therapy should be considered as a subsidiary treatment for acute traumatic cervical spinal cord injury.

\section{References}

1 Kadoya S. Grading and Scoring system for neurological function in degenerative cervical spine disease. Neurosurgical Cervical Spine Scale. Neurol Med Chir (Tokyo) 1992; 32: 40-41.

2 Dituno JF, Marino RJ, Crozier KS. Neurologic and functional assessments in acute spinal cord injury: uses in prognosis and management. Trauma $Q$ 1993; 9: 44-52.

3 Delb W, Muth CM, Hoppe U, Iro H. Outcome of hyperbaric oxygen therapy in therapy refractory tinnitus. HNO 1999; 47: $1038-1045$.

4 Kohshi $\mathrm{K}$ et al. Effects of radiotherapy after hyperbaric oxygenation on malignant gliomas. Br J Cancer 1999; 80: 236241 .

5 Puttaswamy V, Bennett M, Frawley JE. Hyperbaric oxygenation treatment of acute paraplegia after resection of a thoracoabdominal aortic aneurysm. J Vasc Surg 1999; 30: 1158-1161.

6 Veltkamp R, Toole JF. Hyperbaric oxygen - a neuroprotective adjuvant for hyperacute ischemic stroke? J Neurol Sci 1997; 150: $1-2$.

7 Viera C et al. A study of peripheral neural conduction, motor and sensory, in diabetic patients treated with hyperbaric oxygenation. Rev Neurol 1999; 28: 868-872.

8 Hart GB, Thompson RE. The treatment of cervical ischemia with hyperbaric oxygen (OHP). Stroke 1971; 2: 247-250.

9 Oshima $\mathrm{M}$ et al. Effect of oxygenation at high pressure treatment on clinical course and CSF lactate level in acute cerebral infarction. Jpn J Stroke 1988; 10: 208-214.

10 Ishihara $\mathrm{H}$ et al. Prediction of the surgical outcome for the treatment of cervical myelopathy by using hyperbaric oxygen therapy. Spinal Cord 1997; 35: $763-767$.

11 Shin RW, Yagi H, Kusuda K, Oyama M. Efficacy of hyperbaric oxygenation (HBO) therapy for acute or chronic spinal cord lesion. Nichi Koatsu Ishi 1990; 25: 113-117.

12 Tatsumura $\mathrm{T}$ et al. Clinical significance of hyperbaric oxygen therapy (HBO) in the treatment of cerebro-spinal disease. Nichi Koatsu Ishi 1990; 25: 169-176.

13 Gamache Jr FW, Myers RAM, Duker TB, Cowley RA. The clinical application of hyperbaric oxygen therapy in spinal cord injury. A preliminary report. Surg Neurol 1981; 15: 85-87. 